\title{
A high-resolution air temperature data set for the Chinese Tian Shan in 1979-2016
}

\section{Gao et al.}

Correspondence to: Lu Gao (1.gao@ foxmail.com)

The copyright of individual parts of the supplement might differ from the CC BY 4.0 License. 


\section{Supplementary}

Table S1. Elevation of averaged 9 DEM grids and the elevation differences with station elevation (m).

\begin{tabular}{|c|c|c|}
\hline ID & averaged 9 DEM grids elevation & elevation Difference \\
\hline 1 & 1305 & 50 \\
\hline 2 & 306 & 14 \\
\hline 3 & 477 & 2 \\
\hline 4 & 467 & -26 \\
\hline 5 & 764 & 30 \\
\hline 6 & 672 & -9 \\
\hline 7 & 1885 & -34 \\
\hline 8 & 893 & 25 \\
\hline 9 & 2004 & -251 \\
\hline 10 & 1101 & 3 \\
\hline 11 & 868 & 5 \\
\hline 12 & 940 & -18 \\
\hline 13 & 2462 & -4 \\
\hline 14 & 1057 & -2 \\
\hline 15 & 11 & 24 \\
\hline 16 & 1221 & 8 \\
\hline 17 & 978 & -2 \\
\hline 18 & 1066 & 33 \\
\hline 19 & 937 & -5 \\
\hline 20 & 1635 & 3 \\
\hline 21 & 433 & 46 \\
\hline 22 & 1814 & -85 \\
\hline 23 & 758 & -21 \\
\hline 24 & 1548 & 20 \\
\hline Average & & -8 \\
\hline
\end{tabular}


Table S2. Averaged NSE, RMSE and PDF-based skill scores between the original and corrected ERAInterim temperature for different elevation groups.

\begin{tabular}{rcccccc}
\hline & \multicolumn{2}{c}{ NSE } & \multicolumn{2}{c}{ RMSE } & \multicolumn{2}{c}{ PDF-based skill score } \\
\cline { 2 - 6 } & original & correction & original & correction & original & correction \\
\hline $0-500$ & 0.89 & 0.94 & 4.69 & 3.50 & 0.82 & 0.92 \\
$500-1000$ & 0.93 & 0.97 & 3.34 & 2.29 & 0.88 & 0.93 \\
$1000-1500$ & 0.94 & 0.96 & 3.03 & 2.39 & 0.88 & 0.91 \\
$1500-2000$ & 0.85 & 0.94 & 3.61 & 2.55 & 0.86 & 0.90 \\
$2000-2500$ & 0.78 & 0.69 & 6.65 & 7.80 & 0.71 & 0.75 \\
\hline
\end{tabular}


Table S3. Averaged RMSEs of seasonal temperatures between the original ERA-Interim and corrections for different elevation groups.

\begin{tabular}{rcccc}
\hline & Spring & Summer & Autumn & Winter \\
\hline $0-500$ & 1.28 & 1.14 & 1.67 & 4.30 \\
$500-1000$ & 0.93 & 1.37 & 1.12 & 2.04 \\
$1000-1500$ & 0.91 & 1.38 & 1.20 & 2.30 \\
$1500-2000$ & 1.62 & 1.97 & 1.62 & 1.79 \\
$2000-2500$ & 3.22 & 0.42 & 3.23 & 12.80 \\
\hline
\end{tabular}


Table S4 Correlation between observed and modeled lapse rates $\left({ }^{\circ} \mathrm{C} \mathrm{km}{ }^{-1}\right)$ for each month.

\begin{tabular}{ll}
\hline Month & Correlation \\
\hline January & 0.30 \\
February & 0.30 \\
March & 0.38 \\
April & 0.74 \\
May & 0.73 \\
June & 0.49 \\
July & 0.40 \\
August & 0.41 \\
September & 0.60 \\
October & 0.25 \\
November & 0.38 \\
December & 0.36 \\
\hline
\end{tabular}




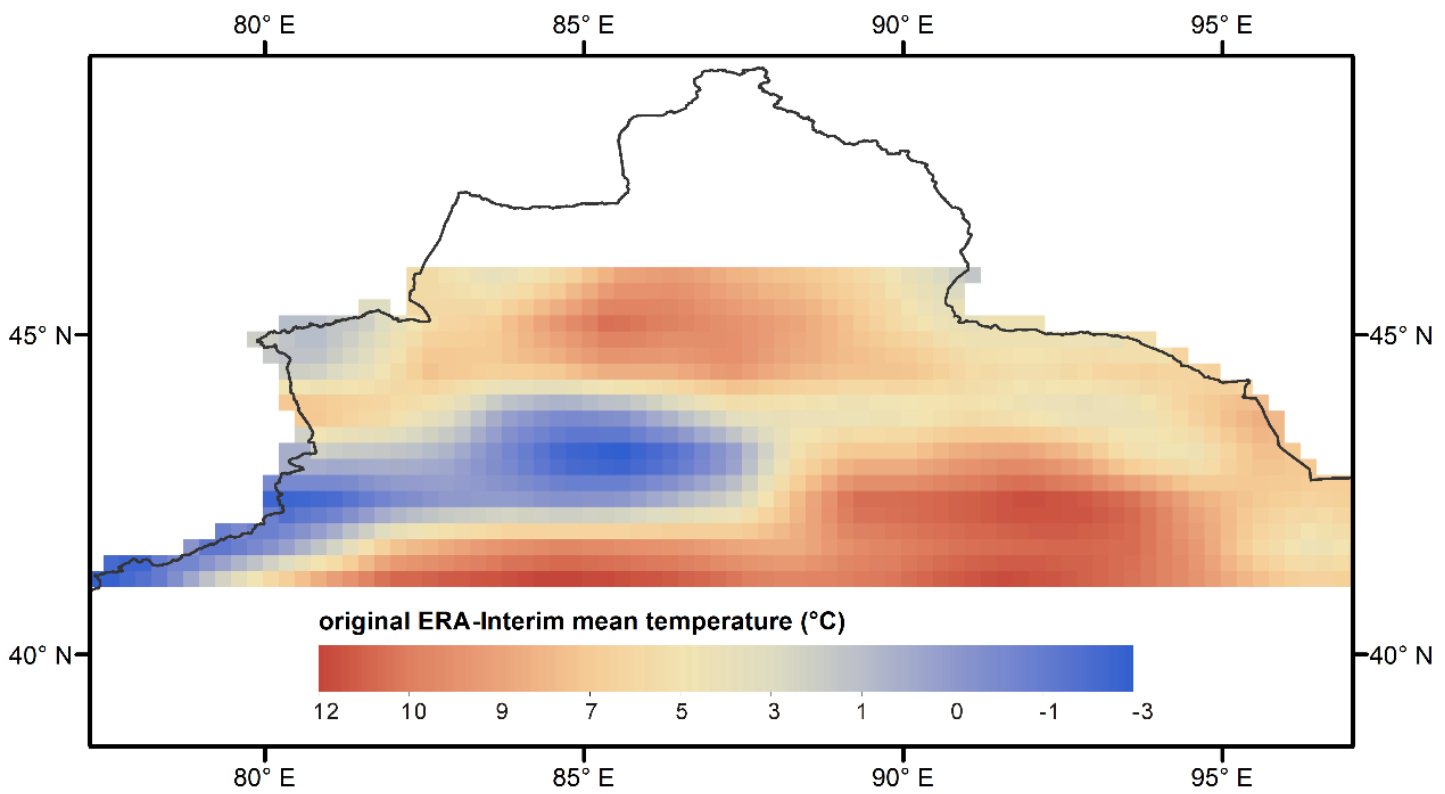

Figure S1: Mean temperature of the entire CTM based on the original ERA-Interim. 


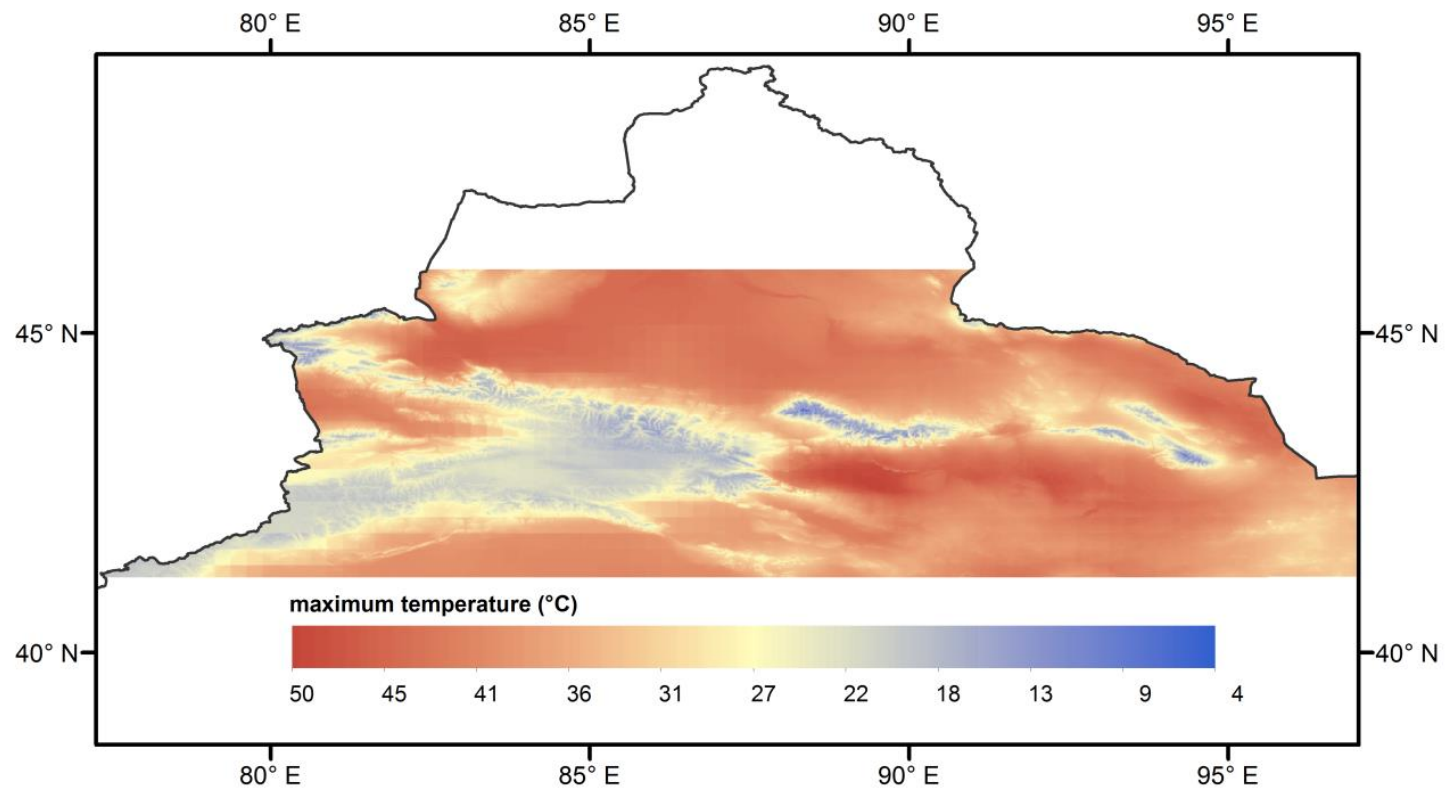

Figure S2: Maximum temperature of the entire CTM based on the high-resolution data set. 


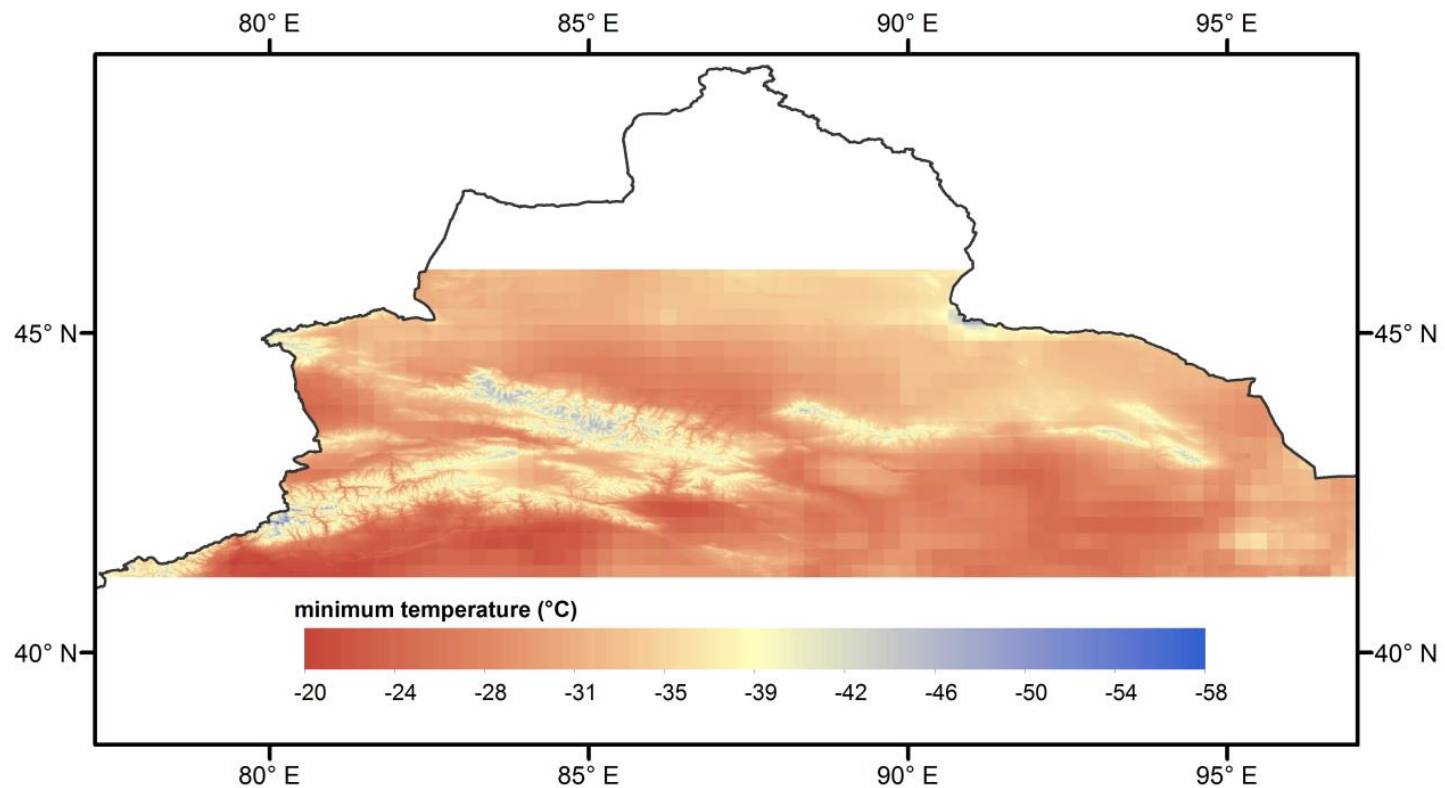

Figure S3: Minimum temperature of the entire CTM based on the high-resolution data set. 